\title{
Intraoperatieve
}

\section{neuromonitoring bij operatie aan de wervelkolom (2)}

In deel 1 (Critical Care nr. 4) van dit artikel zijn het doel, de indicatie en de

methode van intraoperatieve neuromonitoring (IONM) met transcraniële

elektrische stimulatie van motorische evoked potentials (TES MEP) beschre-

ven. In dit tweede deel gaan we in op de vroegere methode, de benodigde

anesthesietechniek en hoe te handelen bij aanwijzingen voor myelumschade

zoals gemeten met TES MEP-monitoring.

Is er vroeger eigenlijk bewaking van het ruggenmerg uitgevoerd? De wake-uptest was de voornaamste bewakingsmethodiek totdat er specifieke monitoring van ruggenmerg en/ of zenuwen werd ontwikkeld. Bij de wake-uptest wordt de patiënt tijdens de operatie wakker gemaakt om op commando de benen en voeten te bewegen. In de regel is de correctie van de wervelkolom dan al gedaan, maar door de anesthesie nagenoeg te staken, wordt de patiënt bijna wakker en kunnen de functies van het ruggenmerg beoordeeld worden. Zodra er bewegingen van de benen en de voeten zijn, is bekend dat het ruggenmerg voor motorische geleiding intact is en wordt de anesthesie hervat en de wond gesloten. Zijn er geen bewegingen tijdens de wake-uptest, dan is neurologische schade bij de patiënt zo goed als zeker. Of er dan nog herstel van de functies zal zijn, hangt af van de chirurgische mogelijkheid om correctie direct te verminderen. De grootte van de neurologische schade wordt dan pas postoperatief duidelijk. Probleem met deze methodiek is dat de 'meting' met de huidige complexe correctiemethodieken pas lang na de correctie plaatsvindt, als de neurologische schade al onherstelbaar is. Vroeger, bij de 'simpele' harringtonstaaf kon direct na de eenvoudige distractie worden gemeten. Bovendien is de betrouwbaarheid beperkt, het is moeilijk te titreren hoe wakker de patiënt is. Sommige patiënten (bijvoorbeeld geretardeerde patiënten) kunnen de opdracht tot bewegen niet uitvoeren. En het is niet bepaald veilig als de patiënt op de ok-tafel met open wond wakker gaat worden en wellicht zo beweegt dat de lijnen verloren gaan.

Vanaf 1985 wordt bij meerdere centra in de wereld somato-sensorische evoked potentials (SSEP)-monitoring uitgevoerd eventueel aangevuld met de wake-uptest (ook met een kans op een vals positieve of vals negatieve uitslag). Deze wake-uptest is met de komst van TES MEP-monitoring in 1993 eigenlijk obsoleet geworden. Daar waar geen MEP-monitoring aanwezig is, kan de wake-uptest nog steeds gedaan worden.

\section{MEP en anesthesietechniek}

Het succesvol monitoren met TES MEP is afhankelijk van een daarbij passende gestandaardiseerde anesthesietechniek. Veel anesthetica hebben een belangrijk deprimerend effect op evoked potentials. Anesthetica zorgen voor een verminderde of zelfs een geblokkeerde prikkeloverdracht bij zenuwcellen; dit leidt bij TES MEP-monitoring tot een zeer grote afname van de amplitude van de responsen. Het beoordelen van de meetgegevens wordt daardoor moeilijk of onmogelijk. Bij het toepassen van motorische evoked potentials is het daarom noodzakelijk om medicijnen die de responsen deprimeren niet toe te dienen of - indien wel nodig - in een dosering waarbij toch gemonitord kan worden.

De medicijnen die tijdens MEP-monitoring vermeden moeten worden zijn: anesthesiedamp (alle gehalogeneerde kool- 


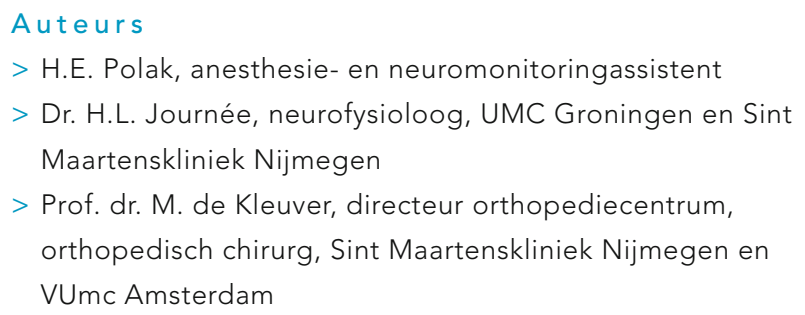

VUmc Amsterdam waterstoffen), relaxantia (depolariserend en niet-depolariserend) barbituraten, benzodiazepines, droperidol, lachgas $\left(\mathrm{N}_{2} \mathrm{O}\right.$ meer dan $\left.65 \%\right)$ en een bolus propofol. Opiaten hebben weinig invloed op de MEP-respons.

De anesthesietechniek waarbij MEP-monitoring goed uitvoerbaar blijft, is totaal intraveneuze anesthesie (TIVA): slaap + opiaat. Zo is bijvoorbeeld de combinatie ketamine of propofol met sufentanil of fentanyl mogelijk. Een anesthesietechniek die ook toegepast wordt, is propofol/ remifentanil/s-ketamine. Na het toedienen van een relaxans voor de intubatie zijn de EMG-responsen laag of geheel afwezig. Na ongeveer 45-60 minuten zullen betere responsen in beeld zijn, zodra een niet-depolariserend relaxans is uitgewerkt.

De bloeddruk en de temperatuur correleren met de trend van de MEP-responsen; dus het is nodig om beide op een juist niveau te houden. Een hoge (normale) bloeddruk geeft peroperatief veel meer bloedverlies uit de grote botwond. Om de bloedstroom in het ruggenmerg (myelum) optimaal te houden en bloedverlies beperkt te houden, wordt daarom een mean arteriële druk van 60-70 mmHg bij jongeren, en $70 \mathrm{mmHg}$ bij ouderen nagestreefd.

Ook de duur van de operatie is van invloed op het bloedverlies. Het is vanzelfsprekend om het circulerend volume stabiel te houden, zeker met deze MEP-monitoring.

Het is tevens belangrijk de patiënt warm te houden, onder andere omdat bij een lage temperatuur de responsen dalen. Daarom moet de kerntemperatuur gemeten worden en zal de patiënt altijd verwarmd worden met één of twee warmeluchtblazers. Wij houden de temperatuur van de patiënt altijd op $\pm 37^{\circ} \mathrm{C}$ ondanks de grote wond, het grote volume toegediende infuusvloeistoffen en de duur van de operatie.

\section{Events tijdens MEP-monitoring}

TES MEP-metingen zijn zeer gevoelig voor verandering in perfusie van het myelum en reageren vrijwel direct met een daling van responsen. Het optreden van een 'event' tijdens de MEP-monitoring betekent dat de gemeten responsen dalen als waarschuwing voor problemen met het functioneren van het myelum. Deze events zijn te verdelen in een responsdaling:
$>$ met een chirurgische oorzaak

$>$ met een anesthesiologische oorzaak

$>$ door een combinatie van de twee bovenstaande oorzaken

$>$ door technische problemen.

Het is noodzakelijk om fysiologische veranderingen te onderscheiden van de pathologische dalingen. Soms is dat eenvoudig, maar dit kan zo met elkaar verweven zijn dat het moeilijk is - of zelfs onmogelijk. Om problemen te kunnen herleiden naar de oorzaak wordt nauwgezet een logboek bijgehouden door de IONM-specialist. Daarin is een mogelijke oorzaak voor een responsdaling terug te vinden en daarmee kan de chirurg ingelicht worden.

Wij hanteren de MEP-amplitudecriteria om daling van responsen te benoemen. Er zijn twee typen dalingen van een respons te onderscheiden: de geleidelijke daling en een abrupte daling.

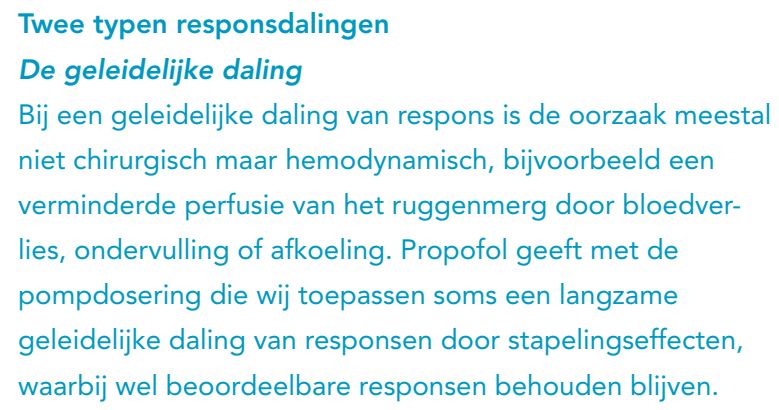

\section{Technische problemen}

Door een technische oorzaak kunnen de responsen plotseling verdwijnen: zo kunnen de stimulatiekabels of de responska- 
bels zijn losgeraakt of stuk zijn, etc. Er is een beslisboom ontwikkeld zodat geen noodzakelijke actie over het hoofd wordt gezien. De volgende stappen zijn dan belangrijk:

$>$ Het waarschuwingscriterium is 70 procent daling van de responsamplitude op 1 of 2 van de 6 meetpunten op de benen.

$>$ Een duidelijke melding van de aard en locatie van de responsdaling wordt aan de operateur gegeven.

$>$ De IONM-apparatuur wordt nagekeken om een technische storing uit te sluiten.

$>$ De stimulatie wordt met een hoger voltage en eventueel met meer pulsen toegediend.

$>$ Bij een blijvende daling van 70 procent of meer is er direct overleg met de chirurg en zo nodig ook met de supervisor van de neuromonitoring over de te nemen actie(s).

$>$ Het anesthesieteam zal mogelijk de bloeddruk moeten laten stijgen d.m.v. vullen, eventueel ondersteund met medicamenten zoals fenylefrine of dopamine.

$>$ De chirurg kan de laatste chirurgische actie (indien mogelijk) tenietdoen.

$>$ Het meest voorkomend om deze situatie te verbeteren, is het verwijderen van één of meerdere pedikelschroeven en/ of het verminderen van de correctie van de stand van de wervelkolom (zie figuur 1).

Wanneer er na deze chirurgische acties en het stimuleren met een hoger voltage en een groter aantal pulsen toch een blijvende responsdaling richting 0 procent gezien wordt, dan is dat sterk suggestief voor myelumschade. Dan zal als laatste redmiddel het verwijderen van de staven (volledige correctie ongedaan maken en spanning van de wervelkolom halen) en het toedienen van een hoge dosering corticosteroïden overwogen worden volgens een vast protocol. In uitzonderlijke situaties kan bij twijfel nog overwogen worden eerst een wake-uptest uit te voeren, maar meestal geeft dit alleen maar onrust en vertraging.

Het snel en adequaat handelen op deze relatief zeldzame situaties (1-5 procent van de operaties, afhankelijk van het type operatie) betekent dat protocollair werken (standaard anesthesietechnieken, standaard protocol wat te doen bij een daling), de ervaring van het gehele team met deze situaties en intensief overleg binnen het team van groot belang zijn om te voorkomen dat de patiënt een (partiële) dwarslaesie overhoudt.
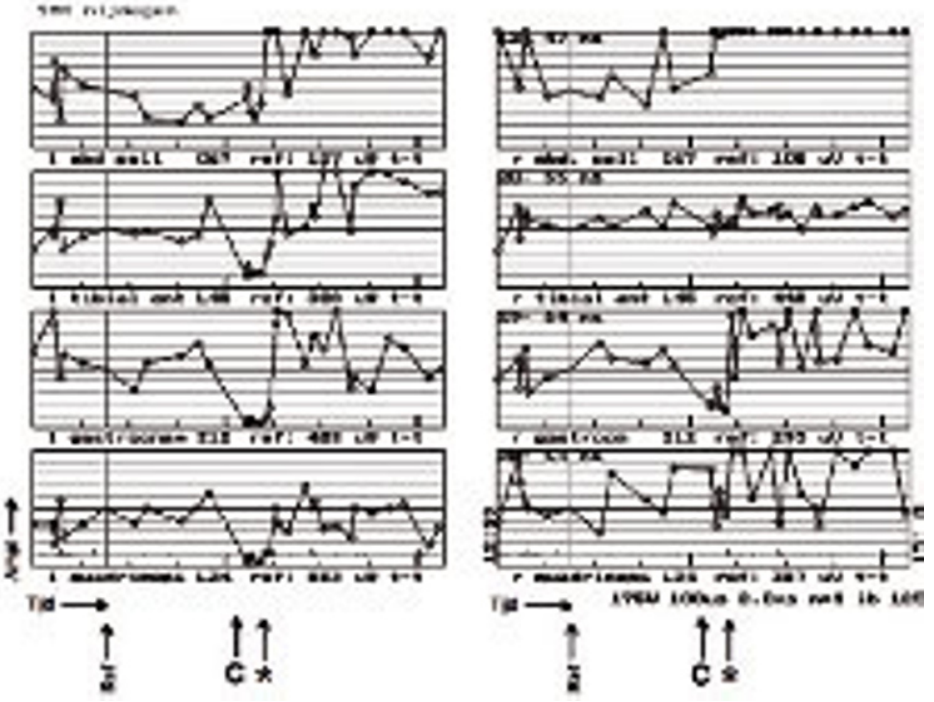

Figuur 1. Trendcurve van de acht spieren tijdens de IONM.

Er is een abrupte daling naar 0 procent van de responsen van de linker beenspieren (M. Tibialis anterior, M. gastrocnemius en M. quadriceps) en de rechter $M$. gastrocnemius naar 30 procent bij TES MEP-stimulatie ten gevolge van de correctie van een scoliose-gemarkeerd met: C. Indien er geen corrigerende actie was geweest, is de kans op blijvende ruggenmergschade met een (partiële) dwarslaesie zeer groot. $\mathrm{Na}$ het losmaken van de staven herstellen de responsen zich onmiddellijk, gemarkeerd met: *. Er is geen schade aan de patiënt ontstaan. De curven geven een beeld van de verhouding van de amplitude van de responsen ten opzichte van het referentie punt. (de verticale lijn). $\mathrm{C}=$ correctie van de scoliose; * ongedaan maken van de correctie; Ref $=$ referentie; Ampl $=$ amplitude

\section{Veiligheid}

TES MEP is zeer veilig: de spiercontractie en de beweging van de patiënt door de stimulatie kunnen in een enkel geval een bijtverwonding veroorzaken van de tong, lip of wang. De endotracheale tube kan ook dichtgebeten worden. Om dit te voorkomen, worden altijd bijtbloks van opgerolde gazen tussen tanden en kiezen geplaatst na de intubatie. Er zijn geen andere relevante complicaties beschreven. Relatieve risicofactoren zijn o.a. een pacemaker en interne defibrillator (storing in de functie van deze geïmplanteerde apparaten zijn in theorie mogelijk, dit is echter nooit beschreven). Epilepsie vormt geen contra-indicatie, de patiënt is immers volledig onder narcose. 


\section{Samenvatting}

Het monitoren van de functie van het ruggenmerg is met TES MEP goed mogelijk. Standaardiseren en afstemmen van de anesthesietechniek op de IONM is een voorwaarde voor goed interpreteerbare monitoring.

Het beoordelen van de curven is complex en vereist kennis van de anatomie en fysiologie van het centrale zenuwstelsel, naast een ruime ervaring in interpretatie van de beelden. Het nauwgezet volgen en het noteren van de operatieve acties en de anesthesiemedicatie zijn noodzakelijk om oorzaken van responsdaling te kunnen herleiden en te communiceren met de chirurg. Een frequente en uitermate heldere communicatie is vereist met de chirurg, anesthesioloog, anesthesieassistent en neurofysioloog. Bewaking van het ruggenmerg (SSEP en/of TES MEP) is onmisbaar tijdens corrigerende wervelkolomoperaties. De wake-uptest moet beschouwd worden als obsoleet. De bewaking met behulp van motor evoked potentials zoals boven beschreven (TES MEP eventueel aangevuld met somato-sensorische evoked potentials) is inmiddels de gouden standaard bij corrigerende wervelkolomoperaties.

\section{Literatuu}

> Behandelrichtlijn scoliose bij neuromusculaire aandoeningen. 2007. Kwaliteitsinstituut voor de gezondheidszorg CBO, Utrecht.

$>$ Calenbergh F van. Editorial Intraoperatieve neuromonitoring. Tijdschr Neurol Neurochir 2010;111 nr 5:188-9.

$>$ Dongen EP van, Beek HT ter, Aarts LP, Schepens MA, Morshuis WJ, Benning FJ, De Boer A, Boezeman EH. The effect of two low-dose propofol infusions on the relationship between six-pulse transcranial electrical stimulation and the evoked lower extremity muscle response. Acta Anaesthesiol Scand 2000; 44: 799-803.

$>$ Hal C van, Hoebink E, Polak HE, Racz I, Kleuver M de, Journée HL. Optimum Interpulse Interval Times in TES elicited MEP Amplitudes for the Upper and Lower extremity Muscle Groups. Lezing op het Symposium Intraoperative Neurophysiologic Monitoring, 2010 Groningen.

$>$ Hoebink EA, van Hal C, Kleuver M de, Racz I, Polak HE, Journée HL. Reducing risk of bite injuries in transcranial electrical stimulation (TES) by optimizing stimulation parameters. Lezing op het Symposium Intraoperative Neurophysiologic Monitoring, 2010 Groningen.

$>$ Jacobs MJ, Elenbaas TW, Schurink GWH, Mess WH, Mochtar B.
Assessment of Spinal Cord Integrity During Thoracoabdominal Aortic Aneurysm Repair. Ann Thorac Surg 2002;74:S1864-6.

$>$ Jacobs J, Janssen H, Hosman AJF, Bartels RHMA, Alfen N van. Scoliose en interoperatieve neuromonitoring. Tijdschr Neurol Neurochir 2010;111 nr 5: 190-9.

$>$ Journée HL, Polak HE, Kleuver M de, Langeloo DD, Postma AA. Improved neuromonitoring during spinal surgery using double-train transcranial electrical stimulation. Medical \& Biological Engineering \& Computing, 2004, 1: 110-113.

$>$ Journée HL, Polak HE, Kleuver M de. Influence of electrode impedance on threshold voltage for transcranial electrical stimulation in motor evoked potential monitoring. Med.Biol.Eng Comput., 2004, 42(4):557-61

$>$ Journée HL, Polak HE, Kleuver M de. Conditioning stimulation techniques for enhancement of transcranially elicited evoked motor responses. Neurophysiol Clin. 2007 Dec;37(6):423-30.

$>$ Langeloo DD, Journée HL, Polak B, Kleuver M de. A new application of TcE MEP: spinal cord monitoring in patients with severe neuromuscular weakness undergoing corrective spine surgery. J.Spinal Disord., 2001, 14, no. 5: 445-448.

$>$ Langeloo DD, Lelivelt A, Journée HL, Slappendel R, Kleuver M de. Transcranial electrical motor-evoked potential monitoring during surgery for spinal deformity - A study of 145 patients, Spine 28, 2003; 1043-1050.

> Langeloo DD, Journée HL, Pavlov PW, Kleuver M de. Cervical osteotomy in ankylosing spondylitis: evaluation of new developments, Eur.Spine J. 15, 2006; 493-500.

$>$ Langeloo DD, Journée HL, Kleuver M de, Grotenhuis JA. Criteria for transcranial electrical motor evoked potential monitoring during spinal deformity surgery A review and discussion of the literature. Neurophysiol Clin. 2007 Dec;37(6):431-9.

$>$ Langeloo DD. Monitoring of the spinal cord during corrective spinal surgery. A clinical study of TES-MEP. Proefschrift. 2007. Nijmegen.

$>$ Polak HE, Racz I, Journée HL, Kleuver M de. Interoperatieve neuromonitoring. NTvAn med. 2009 vol 26; nr5 sep 7-11.

$>$ Scoliosis Research Society: SRS Information Statement, January 2009.

$>$ Ubags LH, Kalkman CJ, Been HD, et al. The use of a circumferential cathode improves amplitude of intraoperative electrical transcranial myogenic motor evoked responses. Anesth Analg 1996; 82:1011-4. 